\title{
Explorando el espacio vial: Un mapeo preliminar de los factores que influyeron en la capacidad de regulación y fiscalización del transporte urbano de la Municipalidad Metropolitana de Lima durante el período 2007-2014 (*)
}

\section{Exploring the urban traffic space. Mapping the factors that affected the regulation and inspection of Lima's urban transport system during the period 2007-2014}

\section{Diego Alonso Sánchez Flórez (**)}

Instituto de Estudios Peruanos

ORCID: 0000-0002-9755-686X

Fecha de recepción: 14 de abril

Fecha de aceptación: 19 de junio

ISSN en línea: $2415-2498$

Sánchez Flórez, D. (2018) «Explorando el espacio vial: Un mapeo preliminar de los factores que influyeron en la capacidad de regulación y fiscalización del transporte urbano de la Municipalidad Metropolitana de Lima durante el período 2007-2014». Politai: Revista de Ciencia Política, Año 9, primer semestre, № 16: pp. 110-140.

DOI: https://doi.org/10.18800/politai.201801.004

* Una primera versión de este trabajo fue presentada en el $9^{\circ}$ Congreso de la Asociación Latinoamericana de Ciencia Política (ALACIP)

** Asistente del Instituto de Estudios Peruanos (IEP). Bachiller en Ciencia Política de la Universidad Antonio Ruiz de Montoya (UARM).dsanchez@iep.org.pe y diegoalonso.sf@gmail.com 


\title{
Sumilla
}

En este artículo se analiza el caso de la Gerencia de Transporte Urbano (GTU) comparando y describiendo los factores que influyeron sobre el desempeño de sus funciones de regulación y fiscalización a lo largo del período 2007-2014, que comprende dos gestiones con orientaciones políticas contrapuestas sobre el sistema de transporte en la ciudad de Lima. Los resultados preliminares dan indicios para confirmar la hipótesis general de investigación propuesta: los principales factores que influirían en la manera en la que se ha venido ordenando el transporte urbano de Lima durante los últimos años son el esquema de gobernanza de la ciudad, el juego político que se han establecido entre las autoridades y las empresas de transporte y las capacidades técnicas y administrativas de la agencia estudiada.

Palabras clave: Sistema de transporte urbano, capacidad estatal, regulación y fiscalización, Lima Metropolitana

\begin{abstract}
This article aims to identify the different factors that have affected the recent urban transport policy of the local government of Lima, through the analysis of the Urban Transport Office (GTU) of the municipality of the metropolitan area of Lima (MML) during the period 2007-2014. The preliminary results emphasize the role of the sectorial and territorial governance of the urban transport, the urban transport stakeholders and the capacities of the governmental local agency dedicated to the inspection and regulation of the transport system in the city.
\end{abstract}

Keywords: Urban transport system, state capacity, regulation and supervision, Metropolitan Lima 
Explorando el espacio vial: Un mapeo preliminar de los factores que influyeron en la capacidad de regulación y fiscalización del transporte urbano de la Municipalidad Metropolitana de Lima durante el período 2007-2014 - Diego Sánchez Flórez

\section{Introducción}

Durante las últimas cuatro décadas en América Latina, el desarrollo y gobierno de las ciudades se ha visto alterado de manera radical por tres procesos simultáneos: la privatización de las empresas que tradicionalmente ofrecían servicios públicos de transporte, saneamiento y vivienda; la creación de nuevas instancias sub-nacionales de gobierno, y acelerados cambios demográficos que han llevado a los países de la región a convertirse en sociedades fundamentalmente urbanas (Figueroa, 2005).

En el contexto de esos tres grandes cambios a nivel regional, el Perú ha sido un caso en el que el estado históricamente ha mostrado más bajas capacidades estatales sobre su territorio (Soifer, 2015). En esa línea, los últimos intentos de reforma al transporte urbano en Lima Metropolitana constituyen un interesante campo de experimentación para estudiar las dificultades que tiene el Estado Peruano a la hora de implementar sus decisiones y de establecer un marco de funciones regulatorias básicas. Todo esto cobra mayor relevancia si se toma en cuenta que este ha sido uno de los sectores que desde décadas ha presentado mayores niveles de informalidad (De Soto, 1989; Burga, 1990).

Este artículo busca identificar los factores que influyeron en el desarrollo de las funciones de regulación y fiscalización del sistema de transporte urbano a nivel sub-nacional durante el período 2007-2014. Con ese fin, se optará por analizar el caso de la agencia estatal encargada formalmente de ordenar el tránsito a nivel sub-nacional: la Gerencia de Transporte Urbano (GTU) de la Municipalidad Metropolitana de Lima (MML). Esta propuesta surge de la necesidad de explicar los cambios y permanencias en el sistema a través del tiempo, los condicionantes del rendimiento de las agencias encargadas de regularlo y el rol que estas han venido cumpliendo más allá de sus atribuciones formales.

En la primera sección de este artículo se realizará una revisión de la literatura que ha analizado el rol de las entidades gubernamentales en la regulación y fiscalización del transporte urbano en la ciudad de Lima. Asimismo, se propondrá un marco teórico que nos permita abordar el estudio de las políticas de movilidad urbana en la actualidad. En la segunda, se expondrá la metodología que se ha elegido para abordar el objeto de 
estudio. Finalmente, se expondrán descriptivamente los hallazgos parciales que fueron encontrados a partir de la aplicación de los instrumentos de investigación.

\section{El rol del Estado en la regulación y fiscalización del transporte urbano de Lima Metropolitana}

\section{a) Las políticas y la gestión del transporte urbano en Lima desde 1930}

Antes de la llegada de las reformas neoliberales, la participación del estado en el transporte urbano de Lima había seguido una trayectoria progresiva en cuanto su nivel de involucramiento formal en el sector. Hasta 1929, predominaba un modelo de «autoridad gendarme» que se limitaba exclusivamente a otorgar autorizaciones de rutas y que mantenía una administración descentralizada a cargo del municipio limeño. Con el inicio de la nueva década, el sector pasa a estar a cargo del gobierno central y se desarrolla un modelo de «autoridad controladora» que enfatiza la regulación y fiscalización de esta actividad. Hacia principios de la década de 1950 se registra un nuevo abanico de atribuciones relacionadas a la «autoridad gestora» que, yendo más allá de la atribución de derechos de explotación y la formulación de reglamentaciones, busca suplir las actividades que, por defecto o deficiencia, los consorcios privados no podían cumplir. A pesar de esto, resulta importante destacar que esta trayectoria no ha tenido una progresión lineal y que, por el contrario, está llena de idas y vueltas que se relacionan directamente a la incapacidad de las autoridades para ordenar el tránsito en términos reales (Burga et al., 1990).

Además de estas atribuciones, el Estado también ha cumplido históricamente las funciones de ordenamiento del espacio de movilidad y formulación de procesos planificación de las políticas urbanas. A lo largo del siglo XX, este tipo de intervenciones en el sector se han caracterizado por ser siempre reactivas al problema del transporte y se han desarrollado a partir de supuestos no realistas con respecto a la ciudad ${ }^{1}$ (Bielich,

\footnotetext{
1 De acuerdo a Bielich (2009) se identifican cinco puntos que ha caracterizado la visión de las autoridades sobre Lima y que, a la larga, han influenciado la forma en la que se ha constituido el sistema vial limeño: 1) el énfasis puesto en el Centro de Lima como punto articulador de la ciudad; 2) la exclusión tácita de ciertas poblaciones, lo
} 
Explorando el espacio vial: Un mapeo preliminar de los factores que influyeron en la capacidad de regulación y fiscalización del transporte urbano de la Municipalidad Metropolitana de Lima durante el período 2007-2014 - Diego Sánchez Flórez

2009:125).

Un factor que permitiría dar cuenta de los vaivenes que ha tenido el gobierno frente a los transportistas - oscilando entre la permisión y la represión- y que también permite explicar la paradoja de un Estado que asume nuevas atribuciones formales mientras no gana capacidad real de ordenar el tránsito sería la naturaleza mercantilista del Estado Peruano. Autores como Burga et al. (1990: 60, 69, 84-85) explican que, a medida que el rol estatal con respecto al sistema fue cambiando, los transportistas ya establecidos comenzaron a utilizar el poder gubernamental para limitar las condiciones de acceso y restringir las posibilidades de competencia. De esta manera, la evolución de las funciones estatales iría en paralelo con la formación de oligarquías dispuestas a aprovechar sus vínculos políticos con el fin de aumentar sus ganancias a partir de la restricción de la competencia y de una burocracia en búsqueda de acentuar su poder mediante pactos que les permitan obtener rentas. De esta forma se explicaría la temprana conformación de un sector informal que opera al margen del Estado para un sector mayoritario de la población.

El trabajo de Bielich (2009: 139-146) nos ayuda a complementar la propuesta de Burga et al. (1990). La autora coincide plenamente con el diagnóstico anterior: históricamente no hubo una actitud clara del Estado frente al transporte urbano. Sin embargo, su explicación no destaca la influencia que puedan haber tenido las organizaciones de transportistas, sino la heterogeneidad del Estado en cuanto a su percepción del problema, su capacidad y voluntad de intervenir en él. Esta sería la razón que estaría detrás de los cambios radicales en las políticas en cortos períodos de tiempo -e incluso la convivencia en un mismo marco temporal de enfoques diferentes sobre el problema- y del fracaso de gran parte de estas iniciativas. La variabilidad de enfoques para enfrentar el problema, de las habilidades técnicas y de determinación para llevarlos a cabo dentro por parte del mismo Estado explicarían que este no haya podido incidir de manera determinante en el sector así como también que los funcionarios públicos hayan mostrado desinterés en cambiar el statu quo debido a los

que se refleja en el hecho que los procesos de planificación no han ido acordes con la explosión demográfica que ha sufrido la ciudad entre las décadas de 1950-1970 y que ha supuesto una masiva urbanización en zonas alejadas del caso urbano; 3) la poca reflexión del Estado sobre su función; 4) el progresivo reconocimiento del carácter realmente existente de la movilidad urbana limeña; 5) la tardía priorización del transporte público y la concepción de este problema en términos de construcción de infraestructura. 
costes políticos que estos implican.

Bajo esta nueva línea argumentativa, la explicación sobre el surgimiento de la informalidad se replantea: se deja de enfatizar la oposición entre el Estado excluyente y los transportistas «piratas» para pasar a resaltar la complementariedad entre ambos. Bielich (2009) entiende que el Estado ha ignorado por largos períodos a este sector sin ponerle obstáculos reales a su funcionamiento - más allá de los ciclos esporádicos de prohibición que han existido- lo que representa un reconocimiento tácito de su relevancia para el sistema. En esta línea puede entenderse radical liberalización del sector mediante el Decreto Ley $\mathrm{N}^{\circ} 651$ en 1991 . Ante sus repetidos fracasos en el sector, el Estado optó abiertamente por dejar de gestionarlo mediante la promulgación de esta normativa (Burga 1990:144).

Por último, es importante mencionar la arquitectura institucional que se ha construido para abordar el tema del transporte urbano en Lima. Burga et al. (1990: 112) mencionan que la constante modificación de las entidades competentes en el sector ha sido una condición permanente en la historia, estos cambios se encuentran íntimamente ligados a la condición antes descrita: a cada ciclo de prohibición, permisión y prohibición corresponde la creación de una nueva oficina gubernamental competente que incorpora nuevas organizaciones prestadoras del servicio y reformula la repartición de beneficios entre ellas y el Estado ${ }^{2}$.

\footnotetext{
2 Un aporte importante en ese sentido, es el registro de las diversas organizaciones que se han encargado del tema. Sin hacer un recuento exhaustivo al respecto, vale la pena mencionar como primer hito el año 1929, cuando el sector pasa definitivamente a ser controlado por el Gobierno Central a través del Ministerio de Gobierno y el de Fomento. Es ahí cuando empiezan a registrarse las primeras iniciativas orientadas a tomar una mayor participación en la regulación del sistema. Un segundo hito lo encontramos en 1963 cuando, ante la crisis generalizada de las empresas tradicionales, el Gobierno Central centraliza definitivamente el control del transporte a lo largo de todo el territorio nacional e inicia las acciones empresariales con fuerza mediante la creación de la APTL en 1965. En esa línea, se reorganiza la Dirección General de Tránsito creando oficinas especializadas para regular ómnibus y colectivos, jefaturas departamentales dependientes y la atribución del control de los tranvías esta oficina. Un tercer hito se da en 1969 con la creación del Ministerio de Transportes dónde la Dirección mencionada es anexada. El cuarto hito corresponde al año 1981 dónde se reestructura el Ministerio y se aprueba un nuevo reglamento sectorial que reconoce - una vez más- a los informales. Por último, hacia 1993 la nueva Constitución transfiere la competencia de regulación a la Municipalidad de Lima tras 60 años de gestión centralizada (Burga et al. 1990:112).
} 
Explorando el espacio vial: Un mapeo preliminar de los factores que influyeron en la capacidad de regulación y fiscalización del transporte urbano de la Municipalidad Metropolitana de Lima durante el período 2007-2014 - Diego Sánchez Flórez

\section{b) La liberalización de 1990 y la gestión del transporte urbano desde un contexto de descentralización}

El final de los ochentas en América Latina trajo consigo la privatización de muchas empresas públicas de transporte, la reducción de la regulación, el recorte de subsidios y un modelo de gestión descentralizado que generó que la movilidad urbana pasara de ser una actividad relativamente organizada - a pesar de su poco alcance - sobre la base de empresas relativamente formales, a abrirse en un abanico de alternativas de movilidad dispersas y atomizadas. La liberalización, sumada a las crisis económicas de 1980 y finales de los noventa, incidió en un aumento de las tarifas, la caída de la demanda por movilidad y en la multiplicación de la oferta a través de la generalización de un nuevo tipo de prestador de servicio informal (Figueroa 2005:53).

Para el caso peruano, todo este proceso se desarrolló en medio de dos coyunturas particulares. De un lado, una progresiva delegación de las funciones de regulación y fiscalización del transporte hacia los municipios provinciales que inicia en 1983 y se fortalece con el proceso de descentralización del 2002 (Bielich, 2009:150-152). De otro lado, un contexto de crisis económica y de radical debilitamiento de las organizaciones de transportistas.

Con la llegada de un nuevo gobierno y la aplicación de medidas de shock a inicios del año 1992, el Estado constituyó el Comité Especial de Promoción de la Inversión Privada encargado de emprender la liquidación del vestigio más saltante de la política estatal anterior en cuanto al transporte: la Empresa Nacional de Transporte Urbano. Apoyado por el BID y el BM, dotado de un presupuesto importante y de un equipo técnico bastante especializado, esta institución inició la reestructuración del sistema de movilidad urbana y marcó la redefinición definitiva del rol del Estado en el sector (Zavala, 1995:116-129).

Bajo este nuevo panorama, tres instituciones quedaron formalmente a cargo del sistema compartiendo atribuciones que nunca quedaron claramente delimitadas: el Ministerio de Transportes y Comunicaciones (MTC), el Gobierno Regional del Callo y la Municipalidad Metropolitana de Lima. Hasta los primeros diez años de la década del 2000, el Ministerio no había 
registrado mayores iniciativas para intervenir en el sector en razón de las restricciones que la nueva Constitución les imponía. En ese marco, los funcionarios del ministerio entendían que su rol pasaba por emitir normativas de carácter nacional y asesorar a los gobiernos sub-nacionales. Por su parte, subsistían serias dudas acerca de las capacidades y la voluntad del Gobierno Regional para regular efectivamente el tránsito (Bielich, 2009:160-162).

Por el lado del municipio limeño, se registra la existencia de una multiplicidad de entidades en su interior con atribuciones en el sector. Específicamente, se destaca la labor de dos: el Instituto Metropolitano del Transporte (Protransporte) y la Gerencia de Transporte Urbano de Lima (GTU). La primera de ellas aparece como un organismo de élite, relativamente más eficiente y planificador. Gran parte de esas virtudes se deben a sus facultades específicas destinadas a establecer y administrar los corredores bus rapid transit (BRT) que están destinados a un espacio delimitado que cubre tan solo el $8 \%$ de la demanda. En ese contexto acotado, la institución maneja cierta autonomía y capacidades para funcionar $^{3}$, que, sin embargo, sólo le han permitido ejecutar esta iniciativa en pequeños tramos sin poder generalizar este sistema de movilidad a toda la ciudad. Por su parte, la GTU destaca como un organismo de línea dedicado a la regulación y otorgamiento de rutas en todo el ámbito metropolitano, desbordada, desorganizada y con pocas iniciativas para el cambio. Esto se comprueba en las falencias que llevaron a la no implementación del Proyecto Integral de Transporte Urbano ${ }^{4}$ que aspiraba a reducir el número de rutas autorizadas y cambiar la mejora de las unidades que brindan el servicio ${ }^{5}$ (Bielich, 2009:156-157).

\footnotetext{
${ }^{3}$ Bielich (2009: 156-157) establece que la mayor capacidad institucional de Protransporte se comprueba en el hecho que, a diferencia de la GTU y el Gobierno Regional del Callao, esta agencia ha contemplado el diseño de planes de operación que plantean esquemas para prevenir las posibles resistencias de los operadores y usuarios.

${ }^{4}$ El Proyecto Integral de Transporte Urbano fue una iniciativa que se trató de poner en práctica en la segunda gestión de Castañeda

${ }^{5}$ Bielich (2009: 156-157) establece que la reducida capacidad institucional de la GTU se comprueba en el hecho que durante el período 2007-2010, esta agencia no pudo programar las medidas necesarias para mitigar las resistencias tanto de los transportistas como a los usuarios que se verían afectados por el Proyecto Integral de Transporte Urbano. Asimismo, esto se corrobora por la mayor desorganización y desconocimiento en cuanto a los plazos de inicio, las exigencias y los nuevos procedimientos a implementarse que se registraron en la misma agencia durante su investigación.
} 
Explorando el espacio vial: Un mapeo preliminar de los factores que influyeron en la capacidad de regulación y fiscalización del transporte urbano de la Municipalidad Metropolitana de Lima durante el período 2007-2014 - Diego Sánchez Flórez

\section{Marco teórico}

El conjunto de investigaciones producidas hasta la fecha sobre el sistema de transporte urbano en Lima Metropolitana permiten identificar los factores más relevantes que han condicionado históricamente el accionar estatal en el sistema de movilidad urbana. En primer lugar, se ha mencionado que una variable importante en el sistema ha sido el esquema institucional que ha gobernado el sector y la ciudad a lo largo de los años y oscilando entre ciclos de (re)centralización y descentralización, en los últimos años (Burga et al., 1990). En segundo lugar, autores como Burga et al. (1990) han destacado el rol de los actores sociales y las pugnas de poder que existen entre ellos y el Estado por afianzar el control y la entrada al sistema lo que ha incidido en ciclos de represión y reflujo del accionar estatal en el sistema. Finalmente, se ha destacado el rol de los técnicos y administrativos -sus imaginarios acerca de la ciudad, sus procesos de planificación, sus prioridades acerca de la mismas- y los recursos con los que cuentan las agencias en las que se desempeñan para poder llevar adelante las funciones que formalmente les exige cumplir la ley con respecto al sector (Bielich, 2009). A continuación, se desarrollarán específicamente estas variables a la luz de tres conceptos que permitirán introducir estos factores a la discusión académica más reciente.

\section{a) Gobernanza}

El primer concepto que se ha utilizado en esta investigación es el de gobernanza entendida como:

(...) El proceso mediante el cual el gobierno, las empresas privadas, las organizaciones de la sociedad civil y los ciudadanos interactúan para definir, acordar y decidir sus principios y objetivos de vida en común y las formas de organización, los recursos y las actividades que se consideran son necesarias y conducentes para realizar los objetivos y principios decididos de interés general [resaltado es del autor]. (Aguilar Villanueva, 2014:12 y 17)

Este concepto se desagrega en tres niveles las actividades constitutivas de la gobernanza. El primer nivel es el de gobernanza pública que hace referencia 
al aspecto directivo del gobierno. En este apartado se encuentran todas las acciones dirigidas a definir la visión y misión general de la función pública que cada una de las agencias públicas debería realizar. El segundo nivel es el de la administración pública y hace referencia a todo el conjunto de acciones que se despliegan dentro de los sistemas administrativos para resolver problemas definidos como «públicos». Por último, tenemos el nivel de la gobernanza corporativa del sector público que hace referencia al manejo de cada una de las entidades administrativas particulares que corren a cargo de funcionarios designados por el poder ejecutivo o legislativo y cuyas habilidades pueden favorecer o impedir una dirección corporativa correcta afectando el desempeño de la administración pública (Aguilar Villanueva, 2014: 25-29).

\section{b) Juegos de poder}

El segundo concepto que usará esta investigación es el de juegos de poder, formulado por Charles E. Lindblom (1991). Por este término se hace referencia al conjunto de interacciones políticas por medio de las cuales los actores políticos buscan ejercer influencia y poder sobre los demás. De acuerdo a la propuesta, dos elementos condicionarían la interacción en cada una de las etapas de la política pública. De un lado, el tipo de actores involucrados. De otro lado, el abanico de mecanismos que estos actores tienen a su disposición para ejercer su control sobre los demás.

Partiendo del presupuesto que, en los sistemas políticos actuales los participantes que actúan de modo inmediato y próximo a las políticas públicas constituyen una ínfima proporción de la población, el autor enfatiza el análisis de los cuatro tipos de élite que intervienen en el proceso de elaboración de las políticas públicas. En primer lugar, se encuentran los cargos públicos designados en las elecciones y los cargos nombrados por estos; En segundo lugar, se identifica a la burocracia encargada de llevar adelante los lineamientos de políticas; En tercer lugar se encuentra la empresa privada cuyo poder deriva de la importancia de las funciones que realizan para la estabilidad económica de la sociedad y los diferentes mecanismos institucionales a través de los cuales los políticos tratan de asegurar el desempeño eficiente de las mismas. Finalmente, se identifican 
Explorando el espacio vial: Un mapeo preliminar de los factores que influyeron en la capacidad de regulación y fiscalización del transporte urbano de la Municipalidad Metropolitana de Lima durante el período 2007-2014 - Diego Sánchez Flórez

los grupos de interés compuestos por personas con gran influencia y expertise en la actividad política y aquellos actores a los que los decisores de política conceden autoridad real (Lindblom, 1991: 77-111).

Por su parte, el autor identifica cuatro mecanismos principales mediante los cuales las diferentes élites políticas ejercen influencia sobre los demás. El primer y más básico instrumento a disposición es la persuasión, ya sea fraudulenta o aquella sustentada en un serio análisis de las perspectivas de pérdidas y ganancias. Un segundo instrumento son las amenazas que se formulan en medio de un entramado institucional que solo faculta a determinados cargos públicos a infligir penas severas en condiciones cuidadosamente circunscritas o a tener el poder de obstruir las iniciativas de otros actores. El tercer mecanismo de control que se menciona es el intercambio. Una primera variedad es el intercambio de favores explícitos. La segunda variedad es la reciprocidad, que crea obligaciones recíprocas indefinidas. El cuarto mecanismo de control es el de la autoridad, el cual implica una relación normalizada de obediencia e incuestionada de un agente hacia el otro debido diversas causas y en el que los métodos de control antes mencionados solo se usan de manera ocasional (Lindblom, 1991: 42-43, 62-67).

Para concluir con esta sección, el autor propone entender que los controles que resultan del uso de estos mecanismos actúan en todas direcciones y que no se ejercen por orden jerárquico sino que el control mutuo se realiza en todas direcciones. Esto nos lleva a plantear un modelo interacción que en vez de ser jerárquico desarrolla esquemas de control recíproco.

\section{c) Fortaleza de las agencias estatales}

Hay un consenso en caracterizar este concepto a partir de los elementos técnico-administrativos y organizacionales de las agencias gubernamentales con las que cuentan estas para llevar a cabo sus tareas potencialmente autónomas. De acuerdo a los autores consultados, los diversos componentes que abarca el concepto de «capacidad» son cuatro: 1) Financiamiento entendido como la disponibilidad y fuentes de recursos económicos y físicos con las que cuentan las agencias gubernamentales para llevar a cabo sus tareas; 2) Captación y administración de recursos humanos, entendido como 
la existencia de procedimientos independientes, estandarizados y meritocráticos de reclutamiento de personal y promoción laboral que ofrezcan oportunidades a los funcionarios de «hacer carrera» en la institución; 3) Profesionalización y especialización entendida como el nivel profesional y cualificación técnica de los funcionarios; 4) Articulación organizacional entendida como la división de tareas dentro de una agencia, su habilidad para coordinar políticas, su nivel de planificación y la manera en la que moviliza sus recursos (Dargent, 2012; Fukuyama, 2004; Skocpol, 1989; Repetto, 2004; Bersch et al., 2013).

Un componente dentro del concepto de capacidad que merece una mención aparte es el de autonomía. Dargent (2012:16) y Bersch et al. (2013:4) identifican que esta se refiere a la ausencia de influencias externas e indebidas en las decisiones de las agencias estatales así como también el aislamiento de las mismas con respecto a intereses y presiones particulares. Los autores resaltan que esta característica alude a una diferenciación del Estado con respecto a otros actores sociales. Además de ello, ambos textos también proponen diferenciar dos componentes dentro de esta variable: la autonomía con respecto a presiones políticas y la autonomía de la burocracia con respecto a sectores de la sociedad.

\section{Metodología}

El objetivo general de este artículo es identificar los factores que influyeron en el ejercicio de las funciones de regulación y fiscalización de la MML durante el período 2008-2014.

Hoy en día en la ciudad de Lima conviven tres sistemas de transporte que son gestionados por diferentes instancias y funcionan con diferentes lógicas y normas. Por un lado, se tiene la Línea 1 del Metro de Lima que constituye la primera etapa del sistema masivo de trenes y es administrado por la Autoridad Autónoma del Tren Eléctrico (AATE) adscrita al MTC. Este sistema da servicio al 3\% de los usuarios de transporte público de la ciudad. De otro lado, se tiene a la Línea 1 del Metropolitano, administrado por Protransporte - Organismo Público Descentralizado adscrito a la MML- y que consiste en un sistema de buses de alta capacidad articulados por 
Explorando el espacio vial: Un mapeo preliminar de los factores que influyeron en la capacidad de regulación y fiscalización del transporte urbano de la Municipalidad Metropolitana de Lima durante el período 2007-2014 - Diego Sánchez Flórez

corredores segregados. Este sistema atiende tan solo al $7 \%$ de los usuarios. Finalmente, se encuentra el sistema regular de transporte urbano de pasajeros, administrado también por la Municipalidad a través de la GTU (órgano de línea que depende del presupuesto que le asigne la comuna) y que atiende a cerca del $90 \%$ de los usuarios de transporte público de la ciudad (Glave, 2016: 53).

Desde esta perspectiva, el análisis de la GTU es especialmente relevante para esta investigación dado que se trata de una agencia que concentra todas las dificultades posibles para poder ejercer adecuadamente sus funciones $\mathrm{y}$, dada esta cualidad, reúne a través de su estudio todos los factores que han estado influyendo en el desarrollo de las políticas de transporte urbano.

Por otro lado, es importante destacar que esta investigación centrará su atención en analizar el período 2007-2014 que corresponden a los gobiernos de los alcaldes Luis Castañeda Lossio (2007-2010) y Susana Villarán (20112014). Aunque ambas gestiones se desarrollaron en una etapa en la que se registran por parte del Estado un conjunto de acciones que intentan reordenar el sistema de movilidad urbana, es importante destacar que cada una desarrolló políticas con respecto al transporte con prioridades diferentes (Glave, 2016: 56-66). En esa línea, analizando diferentes tipos de políticas de transporte urbano, esta selección temporal nos permitirá identificar mejor la diversidad de variables que intervienen en el accionar estatal del sector.

La presente investigación tiene un enfoque cualitativo. La data referida al accionar de la GTU durante el período 2008-2014 se obtuvo por medio de revisión de fuentes secundarias y cinco entrevistas semiestructuradas realizadas a exfuncionarios de la Municipalidad que trabajaron durante el período referido. Las documentos consultados fueron dos informes de gestión formulados durante el período que cubre este estudio: «El Transporte Urbano en Lima Metropolitana: Un desafío para la defensa de la vida» publicado en el 2008 por la Defensoría del Pueblo y «Lima lo hizo: Informe de gestión 2001/2014» publicado en 2014 por la misma MML.

Por su parte, con respecto al perfil profesional de los entrevistados, es importante destacar que todos ellos cumplieron cargos directivos o puestos de asesoría técnica en alguna de las dos gestiones dentro del Municipio. En la siguiente tabla, se especificará los cargos y las agencias donde se desenvolvieron. 


\begin{tabular}{|c|c|}
\hline Agencia & Cargo \\
\hline $\begin{array}{c}\text { Gerencia de Transporte } \\
\text { Urbano-Sub-gerencia de } \\
\text { Regulación y Fiscalización del } \\
\text { Transporte }\end{array}$ & Sub-gerente \\
\hline Gerencia de Transporte Urbano & Asesor legal \\
\hline Protransporte & Miembro del directorio \\
\hline $\begin{array}{c}\text { Gerencia de Promoción de la } \\
\text { Inversión Privada }\end{array}$ & Asesor de proyectos \\
\hline $\begin{array}{c}\text { Gerencia de Transporte Urbano } \\
\text { Consultor técnico en temas de } \\
\text { emisión de licencias de } \\
\text { conducir, licitaciones y retiro de } \\
\text { unidades de transporte }\end{array}$ \\
\hline
\end{tabular}

Cuadro 1. Actores entrevistados

\section{Hallazgos parciales de investigación}

El objetivo general de esta sección es sostener la hipótesis inicial de que los factores que han afectado las políticas de movilidad urbana están vinculados al esquema de gobernanza del sector y la ciudad, a la influencia de los juegos de poder que se han establecido entre actores políticos y sociales y al conjunto de recursos administrativos y políticos con los que contaban las agencias estatales de transporte. Al momento de definir los principales problemas que el sistema de transporte urbano enfrenta, todas las fuentes consultadas — tanto los documentos de gestión como los resultados de las entrevistas - coincidieron en un conjunto de elementos que podemos ordenar en cuatro ejes diferentes. 
Explorando el espacio vial: Un mapeo preliminar de los factores que influyeron en la capacidad de regulación y fiscalización del transporte urbano de la Municipalidad Metropolitana de Lima durante el período 2007-2014 - Diego Sánchez Flórez

\section{a) Diseño del sector transporte urbano}

Con respecto a este eje, lo primero que destaca es la enorme dispersión con la que formalmente se han asignado funciones y jurisdicciones dentro del sistema de transporte urbano de Lima (Defensoría del Pueblo, 2008: 164). Las entrevistas realizadas arrojaron que en la actualidad existen un total de 53 instituciones divididas en tres niveles de gobierno que tienen algún tipo de competencia formal o algún grado de injerencia en el sector y que, dadas las diversas lógicas que los guían, complejizan mucho la tarea de llevar adelante la implementación de políticas públicas en el tema. Este panorama se puede comprender mejor si se tiene en cuenta que el transporte tiene tres grandes áreas de especialidad: el diseño y construcción de infraestructura vial, el control del tránsito y la movilidad y la regulación de los modos de transporte. Bajo este panorama, se ha conformado en la práctica un esquema de división funcional que en el que no queda definido con claridad a qué nivel de gobierno le compete cada tipo de actividad. Asimismo, a esta situación se le añade la división político-territorial de la ciudad de Lima que está dividida en dos jurisdicciones diferentes - Lima Metropolitana y Callao- a pesar de constituir una misma metrópoli y, al interior de cada una, sus autoridades provinciales y regionales están fraccionadas en una multiplicidad de autoridades distritales.

Gran parte de la que se ha descrito puede explicarse a partir de tres procesos, aunque se encuentran íntimamente vinculados entre sí, es importante diferenciar adecuadamente. En primer lugar, el actual diseño institucional de las municipalidades en el país. Este tiene su antecedente inmediato en la promulgación de la Constitución de 1993 que, a diferencia de su predecesora no estableció competencias específicas para las municipalidades provinciales ni les otorgó preeminencia formal o de facto $^{6}$ sobre los municipios distritales. En segundo lugar, la forma en la que se llevó adelante la última reforma de

\footnotetext{
${ }^{6}$ Hubo varios mecanismos a través de los cuáles en la práctica se restó poder a los gobiernos provinciales bajo el fujimorismo: 1) La creación del Foncomún que estableció criterios de distribución de recursos que asignaban más recursos a los municipios más pequeños; 2) La transferencia formal de competencias hacia las municipalidades distritales (administración del Vaso de Leche, parques zonales y habilitaciones urbanas); 3) La interferencia directa del gobierno central en temas como el transporte urbano de Lima Metropolitana; 4) La permanencia de importantes vacíos legales acerca de los mecanismos de coordinación adecuados entre municipalidades provinciales y distritales (Muñoz, 2005: 41, 43, 44 y 47).
} 
descentralización bajo la cual, se desaprovechó la oportunidad para poder resolver el problema que se generaba al contar con dos niveles de gobierno local con las mismas competencias y cuyas relaciones no se encontraban adecuadamente reguladas $^{7}$ (Muñoz, 2005: 37, 52, 59 y 60). En tercer lugar, las transferencias de competencias desde el gobierno central a los gobiernos sub-nacionales que se desarrollaron a partir de la implementación de la reforma descentralizadora y que, en medio de la enorme indeterminación que generaba el marco legal, abrió espacio para el proceso se desarrollara de manera arbitraria e incompleta.

Dentro del nivel nacional, los entrevistados identificaron cuatro ministerios con algún tipo de injerencia en el sector. El primero de ellos es el Ministerio de Transportes y Comunicaciones (MTC), que formalmente es el ente rector del sector. Como tal, esta institución tiene la potestad de elaborar y modificar los dos reglamentos principales que rigen el transporte urbano: el Reglamento de Administración de Transporte, que regula la calidad de servicios de transporte inter-urbano y urbano, y el Reglamento de Tránsito, que regula el control de la velocidad y todas las normas de tránsito. Asimismo, a través de la Dirección General de Caminos y Carriles, este ministerio es el encargado de elaborar las normas técnicas sobre trenes urbanos.

Aunque formalmente el MTC debe asumir de acuerdo a ley la rectoría del transporte urbano en todas las funciones que esta actividad compromete (infraestructura, transporte y tránsito), un entrevistado manifestó que, en la práctica, durante estos años se han registrado acciones que manifiestan cierta reticencia del mismo por asumir este rol $^{8}$. Por el contrario, otro entrevistado

\footnotetext{
${ }^{7}$ Bajo el marco legal actual, lo único que existe son disposiciones puntuales para resolver el problema (establecer la participación de los alcaldes distritales en los Consejos de Coordinación Provincial) y otras de carácter técnico (la exigencia de que los planes de desarrollo distrital se adecúen al provincial). Bajo ese panorama, la asignación de competencias y funciones entres los tipos de municipalidad y se observan superposiciones (Muñoz, 2005: 59$60)$.

8 «(...)Pero creo que el Ministerio se ha caracterizado por no ejercer su rectoría. Porque es el órgano rector, usted es que dicta los lineamientos económicos, organizacionales, las líneas matrices, las políticas de Estado en materia de transporte. Más bien el Ministerio, lo que he notado en todos estos años es que ha sacado cuerpo en todos estos años y no se ha querido involucrar con estos temas de transporte urbano. No ha atacado los problemas estructurales del transporte. Siempre ha sido un Ministerio, digamos, dependiente de otros. Con poca iniciativa. Las Municipalidades tienen un problema en general de falta de institucional, falta de capacidades técnicas, falta de recursos, que también las ha llevado a colapsar.» (Exconsultor de la MML en temas de retiro de unidades, emisión de licencias de conducir)
} 
Explorando el espacio vial: Un mapeo preliminar de los factores que influyeron en la capacidad de regulación y fiscalización del transporte urbano de la Municipalidad Metropolitana de Lima durante el período 2007-2014 - Diego Sánchez Flórez

identificó que sí existirían claras señales de que, esta entidad gubernamental habría priorizado el desarrollo de medidas que —en razón de favorecer la gran inversión privada en la ciudad- trascienden su competencia y que buscaban empujar a los municipios a actuar en la liberación de predios ${ }^{9}$.

Además del rol promotor, en los últimos años, esta institución ha asumido de facto la administración del sistema del tren eléctrico de la ciudad a través de la Autoridad Autónoma del Tren Eléctrico (AATE). Esta institución está a cargo de preparar los estudios para poder poner adelante las líneas de metros y también supervisar los contenidos de concesión cuando los hacen por obra pública. La historia de la manera en la que el gobierno central obtuvo esta competencia factual es larga y comprende un conjunto vaivenes y pugnas con la Municipalidad Metropolitana de Lima (MML) que se remontan a la década de los ochenta ${ }^{10}$.

Un segundo actor relevante el sistema es el Ministerio de Economía y Finanzas (MEF) que cumple un rol en la construcción de infraestructura vial y urbana a través de la Agencia de Promoción de la Inversión Privada (Proinversión) organismo público ejecutor adscrito a dicho sector con autonomía técnica, funcional, administrativa, económica y financiera. Cuando un proyecto de transporte grande - como es el caso del tren- que involucra licitaciones se hace por inversión privada esta institución también se ve involucrada en el esquema.

\footnotetext{
${ }^{9}$ En palabras de un exfuncionario de la GPIP: «Por ejemplo un conflicto ahorita que está sucediendo, no conflicto sino un problema es en la carretera central. La Línea 2 del metro ha propuesto que en ciertos tramos donde están las estaciones, eliminar las vías auxiliares ¿Ya? Que se cierren y que se hagan veredas anchas. Como idea está bien, pero como realidad, atrévete tú como municipalidad provincial que le vas a cerrar el acceso a toda la gente que tiene comercio en su frente, que tiene derechos adquiridos, que ha hecho sus estudios, ha ganado, todo un montón de cosas y que mañana tú le quites eso, eso es primero un impacto social (...) La municipalidad no tiene recursos para construir tren, no tiene. El único que tiene capacidad de construir trenes en este país es el Ministerio. O sea, el ministerio tiene la plata pero no las funciones ni el problema. La municipalidad tiene las funciones y el problema pero no tiene la plata.» (Ex funcionario de la GPIP)

10 «Lo que ocurre es que inicialmente Jorge del Castillo trató de hacer el tren eléctrico (...) luego, como el Ministerio al principio era muy débil, esa instancia fue transferida al MTC y el MTC asumió esa responsabilidad. Luego, como el gobierno de transición [se] consideró que el tren urbano era un tema de la Municipalidad, le dio esa competencia al municipio de Lima en la época de Andrade, me parece. Luego, Castañeda dijo que haría un tren y que estaba en contra del metropolitano, que era una propuesta de Andrade, originalmente y ganó las elecciones con el mensaje de "El tren contra el Metropolitano" pero luego se dio cuenta que no podía con el tren y se lo devolvió al Ministerio de Transportes. Al final Castañeda sí ejecutó el metropolitano, que dijo que no se debía hacer (...)» (Exfuncionario de Protransporte)
} 
Por su parte, los entrevistados identificaron que el Ministerio de Vivienda (Vivienda) es otro actor relevante en el sistema. Específicamente, un exfuncionario mencionó que su rol se ha centrado en también en la función de construcción de infraestructura vial y urbana, solo que ya no en calidad de promotor de la inversión privada, sino en calidad de supervisor de las obras urbanas formuladas por las municipalidades. En ese sentido, se entiende que aunque el MTC tiene la rectoría formal sobre el sector, Vivienda tiene injerencia directa en el mismo ya que es el encargado de aprobar los reglamentos de edificación urbana para calles, ciclovías, veredas y vías urbanas a través del Reglamento Nacional de Edificaciones ${ }^{11}$.

El cuarto actor involucrado es el Ministerio del Interior (Miniter) que cuenta con competencias en la función de control y fiscalización del tránsito de la ciudad través de la Policía. En ese sentido, el rol que ha venido ejerciendo este sector no es normativo ni promotor sino mucho más operativo. Aunque la coordinación y rectoría de la movilidad urbana está a cargo de los municipios provinciales estos dependen de los efectivos policiales para efectuar las sanciones e incluso estos últimos puede intervenir en el sistema semafórico en determinadas circunstancias que ellos consideren necesarias

\footnotetext{
11 «[Las competencias de los municipios provinciales y distritales en materia de infraestructura vial y urbana] En ambos casos [se encuentran] en el marco de los reglamentos de edificación que saca el Ministerio de Vivienda. Entonces un municipio actúa en el marco del gobierno [como] rector de transporte para la calidad urbana pero la norma técnica relevante para los fines [de construcción] es la norma técnica de vivienda (...) Para las normas técnicas [de trenes] es el Sistema de Trenes Eléctricos y Urbanos y para todo lo que es calle y ciclovías, como te acabo de mencionar, y veredas, vías urbanas, en general, la norma técnica la emite el Ministerio de Vivienda que es el Reglamento Nacional de Edificaciones». (Exfuncionario de Protransporte)
} 
Explorando el espacio vial: Un mapeo preliminar de los factores que influyeron en la capacidad de regulación y fiscalización del transporte urbano de la Municipalidad Metropolitana de Lima durante el período 2007-2014 - Diego Sánchez Flórez

como las «horas punta» ${ }^{12}$.

En el nivel sub-nacional, como ya se mencionó, tanto la MML y la Municipalidad Provincial del Callao - como municipalidades provincialesy el Gobierno Regional del Callao tienen facultades en el sistema dado que son, de acuerdo a la ley, los entes rectores del tránsito en sus territorios teniendo la potestad de dictar los lineamientos de las rutas y con responsabilidad para el tema de infraestructura vial urbana. De otro lado, se encuentran los municipios distritales, que en Lima ascienden a 43 y en el Callao a 06 distritos, que tienen un papel en la edificación de calles y la señalización. Este solapamiento de atribuciones ha generado en la práctica serias dificultades para que la MML pueda centralizar, o al menos establecer criterios mínimos, en el ejercicio de las competencias de control del tránsito y edificación de infraestructura vial. Asimismo, contribuye a generar un espacio de movilidad radicalmente segmentado ${ }^{13}$.

Además de ello, las municipalidades provinciales son las instancias encargadas de elaborar los planes de movilidad y los planes urbanos. Los exfuncionarios entrevistados explicaron que subsisten problemas graves porque ocurre que muchas veces los planes urbanos y de movilidad no siempre dialogan entre sí, en el mejor de los casos. En otras ocasiones la

12 «(...) Luego, la policía tiene una función a nivel operacional. Si bien el gobierno regional es el ente rector de tránsito en su provincia, la policía tiene un rol operacional que le puede permitir que, si ellos creen que el ciclo semafórico computacionalmente establecido por un municipio está mal en hora punta, lo pueden cambiar. Operacionalmente, la policía de tránsito opera como si tuviera autonomía pero en estricto aún están bajo la dirección, coordinación y rectoría de cada gobierno provincial. Para el tema de sanciones, tanto en tránsito como en transporte, los municipios emiten las papeletas, se las dan a los policías, los policías sancionan y hay un acuerdo en el reparto de la plata: $70 \%$ municipios, $30 \%$ policías, 75,25 , hay varias formas. Aún en el transporte interprovincial cada municipio regula las carreteras y debe darle las papeletas a la policía de carreteras para que sanciones, y en caso de las sanciones de transporte, porque en transporte tú tienes el Reglamento de Servicio de Transporte Nacional y debajo las ordenanzas que regulan en cada provincia; esas ordenanzas a veces tienen la Tabla de Sanciones que a veces están en otra ordenanza que definen las sanciones. Esas sanciones que son de transporte, no de tránsito, solo la pueden poner los inspectores municipales (...)» Exfuncionario de Protransporte

13 «Todo el mundo se pregunta por qué sólo hay ola verde en los semáforos de la Petit Thuoars y de la Arequipa, Arenales. La razón es muy sencilla, porque la Municipalidad Metropolitana de Lima, porque la Municipalidad de Lima no puede meterse en los proyectos de semaforización de Lince, del distrito de Magdalena, de Surco. Cada quién contrata sus diversos sistemas semafóricos, con su propia tecnología.» Exconsultor de la MML en temas de retiro de unidades, emisión de licencias de conducir. 
situación empeora dado que no siempre estas instancias formulan o actualizan estos documentos de gestión.

Sintetizando lo anterior, se aprecia que las autoridades provinciales y distritales comparten competencias en el desarrollo infraestructura teniendo que seguir la normativa de dos ministerios distintos (MTC y Vivienda). Las provincias participan en la construcción de vías expresas, arteriales y colectoras mientras que los distritos tienen competencia para infraestructura en vías locales que incluyen ciclovías. Por su parte, en el control del tránsito la competencia recae exclusivamente en los municipios provinciales, inclusive para vías locales, pero para desarrollar esta función se necesita, una vez más, el apoyo del gobierno central a través de la Policía y muchas veces también de los municipios distritales. Con respecto al modo de transporte ocurre otro conjunto de traslapes. Las municipalidades provinciales tienen la competencia en la definición y regulación de los modos de transporte, aunque ya vimos que esta función también ha sido recortada en la práctica cuando el MTC asumió el control del Tren Eléctrico, salvo en los mototaxis cuya regulación es competencia del distrito del área donde se desenvuelven. Asimismo, las autoridades provinciales también tienen que someterse al sistema de inversión pública regido por el MEF en caso necesiten realizar alianzas conjuntas con el sector privado para licitaciones de gran envergadura.

Uno de los grandes problemas que trae este sistema es que muchas veces las funciones establecidas para cada nivel de gobierno no tienen correspondencia con el nivel de capacidades y recursos que tienen y, por el contrario, muchas de las entidades que tienen los recursos necesarios, no necesariamente cuentan con las atribuciones formales ni están cerca de las demandas de los usuarios más afectados ${ }^{14}$.

Tres puntos se destacan de manera implícita de las respuestas de los entrevistados en este punto. En primer lugar, la multiplicidad de vínculos que

\footnotetext{
14 Un caso ejemplar en ese sentido fue la construcción de la Línea 1 del tren eléctrico. En palabras de un exfuncionario: "El problema...quien tiene la capacidad de construir tren no es la municipalidad. La municipalidad no tiene recursos para construir tren, no tiene. El único que tiene capacidad de construir trenes en este país es el Ministerio. 0 sea, el ministerio tiene la plata pero no las funciones ni el problema. La municipalidad tiene las funciones y el problema pero no tiene la plata" Exfuncionario de la Gerencia de Promoción de la Inversión Privada.
} 
Explorando el espacio vial: Un mapeo preliminar de los factores que influyeron en la capacidad de regulación y fiscalización del transporte urbano de la Municipalidad Metropolitana de Lima durante el período 2007-2014 - Diego Sánchez Flórez

existen entre el gobierno nacional y los municipios. No se trata de un tipo de vínculo estandarizado sino, antes bien, una amplia variedad que cubre relaciones jerárquicas y de rectoría y supervisión, otras vinculaciones más operativas y horizontales e intervenciones esporádicas para grandes licitaciones y situaciones no reguladas que estos varían de acuerdo a las funciones que se van a cumplir y al tipo de sector con el que se tiene contacto. Otro punto a destacar es que muchas veces estos vínculos involucran al nivel distrital y el nacional sin que medie ni siquiera el nivel provincial o regional socavando la posibilidad de que las funciones del transporte urbano se centralicen en un nivel intermedio de gobierno.

A esta extrema dispersión en la cantidad de instituciones involucradas en el sistema y de vinculaciones poco regularizadas entre niveles de gobierno identificada por los exfuncionarios se le añade una historia de fracasos a la hora de establecer organismos de coordinación intergubernamental. En ese sentido, a lo largo de estos años se registra la sucesión de diversas instancias que apuntaban a ser plataformas de cooperación entre la MML, el Callao y el MTC y que, sin embargo no lograron consolidarse en el tiempo: el Comité de Transporte Metropolitano de Lima (TRANSMET) creado en el $2003^{15}$ y la Comisión Tripartita creada en el $2007^{16}$ (Defensoría del Pueblo, 2008: 79-81 y 164-165).

\section{b) Marco normativo}

En este eje, los entrevistados y los informes de gestión destacaron el notable impacto que tuvo la política de desregulación de la década de 1990. Aunque el aspecto más visible fue la liberalización de las rutas, es importante recordar que esta incluyó otras dos iniciativas. De un lado, la libre importación de vehículos usados. Medida que estuvo justificada en un primer momento por la notable escasez de unidades de transporte pero que, sin embargo, sin justificación técnica aparente permaneció en nuestro marco legal hasta el 2012 y afectó a la competencia automotriz legal. Por otro lado,

\footnotetext{
${ }^{15}$ Esta instancia fue creada como un espacio de cooperación entre la MML y el gobierno central para la gestión de las inversiones en la ciudad y cuyo órgano directivo está compuesto por las máximas autoridades del Instituto Metropolitano de Planificación (IMP), Protransporte, GTU, Empresa Municipal Administradora de Peaje (EMAPE), Fondo Municipal de Inversiones (Invermet) y la AATE.
}

${ }^{16}$ Esta instancia fue creada como un espacio de cooperación entre la MML, la Municipalidad Provincial del Callao y el MTC como un espacio de cooperación para el tema de tránsito y rutas compartidas entre las dos provincias. 
la flexibilización en los requisitos para el otorgamiento de licencias de conducir que se llevó adelante como una medida paliativa con el fin de poder reubicar a todos los trabajadores que salían del sector público y privado ${ }^{17}$. Estos tres elementos combinados cambiaron en pocos años las características existentes del sistema hasta ese momento: de un notable déficit se pasó a una exacerbada sobreoferta de unidades — poco adecuadas además para el servicio y con muchos años de antigüedad- y a la una saturación de las rutas a nivel metropolitano (Defensoría del Pueblo, 2008: 58-60 y Municipalidad Metropolitana de Lima, 2014: 41).

De acuerdo a lo que han explicado los funcionarios entrevistados, se pueden identificar tres impactos principales derivados de estas reformas. El primero de ellos fue el corte exabrupto que significó la política de liberalización de inicios de los noventa para el proceso de aprendizaje de los municipios en cuanto a la regulación del sector ya que, en la práctica, lo que hizo esta medida fue quitarle cualquier prerrogativa que pudieran tener las instancias públicas (nacionales o subnacionales) sobre el transporte urbano. Comparando lo expuesto con los aportes de Glave (2016) podemos apreciar que es recién a partir de la segunda gestión de Andrade (1998-2002) que este proceso da serias señales de comenzar a revertirse. Viéndolo en perspectiva, se tiene un lapso de quince años desde que las políticas de regulación de los modos de transporte recién puedan ser efectivamente ejercidas por la MML.

Un segundo impacto relevante $\mathrm{y}$, que hasta el momento no ha podido revertirse en lo absoluto, es que, de acuerdo a los Decreto Legislativo $\mathrm{N}^{\circ} 651$, el transporte deja de ser catalogado a nivel nacional como «servicio

\footnotetext{
17 «Bueno, yo diría que los tres elementos distintivos de la década más protagónica, o más relevante en cuanto a las decisiones estatales, en materia de transporte ha sido en los 90. No precisamente para bien, sino para mal. (...) Dentro de esa concepción del estado no regulador, se dieron algunas medidas de transporte que fueron lapidarias para el país. Uno la desregulación, los dispositivos, como el control legislativo 640,651, los dos de transporte interprovincial y transporte urbano, que decretaron la libertad de rutas, tanto provincial como urbano. (...) Sin embargo, esa medida de desregulación de las rutas, estuvo acompañada de otra medida, también funesta. Que quizá podía haber justificado por unos meses o por un año, sin embargo duró hasta el año 2012. Que es la liberalización de importación de vehículos usados, y los famosos talleres de cambio de timón. (...) Además se exigía pocos requisitos la licencia de conducir, se exigía secundaria completa para manejar un bus urbano, y para facilitar trabajo para todos los despedidos del sector privado y púbico que entraban al transporte se cambió el secundaria completa, por un simple sabe leer y escribir. (...)» Exconsultor de la MML en temas de retiro de unidades, emisión de licencias de conducir.
} 
Explorando el espacio vial: Un mapeo preliminar de los factores que influyeron en la capacidad de regulación y fiscalización del transporte urbano de la Municipalidad Metropolitana de Lima durante el período 2007-2014 - Diego Sánchez Flórez

público». Esta condición del sector elimina un conjunto de herramientas legales que pueden ser utilizadas por las autoridades para poder mejorar el control y el servicio, tal y como ocurre con el agua y la electricidad, en las que pueden utilizarse subsidios o fijación de tarifas. Esta situación es crítica si se tiene en cuenta las características específicas del sector de transporte urbano que requiere de algún tipo de subsidio que permita invertir en la compra y operación de una flota lo suficientemente grande como para cubrir la totalidad de la demanda en horas punta ${ }^{18}$.

Una tercera consecuencia de este proceso es la entrada vertiginosa de un nuevo actor en el sistema que no había podido desarrollarse plenamente en los esquemas de regulación anteriores del sector: el sector privado. Esta pasa a convertirse en un poderoso elemento que, por un lado, influye en la dirección que van a tomar los gobiernos municipales en el transporte urbano. En ese sentido, no parece ser una coincidencia que las iniciativas consideradas más exitosas en la materia o aquellas en las que se ha puesto mayor prioridad - llegando incluso a generar disputas con el gobierno central- se hayan realizado en las pocas áreas en las que se ha podido introducir a este actor dentro del sistema ya sea a través de la construcción de grandes obras infraestructura vial y licitaciones diversas. Por su parte, precisamente en las áreas donde predominan empresas con menor capital y menor posibilidad de que la gran inversión entre - como es el caso del transporte regular - es donde se registra menos interés o menos capacidad para iniciar acciones en ese sentido ${ }^{19}$. Por otro lado, la entrada del sector privado al sistema también ha abierto todo un conjunto de tareas vinculadas a su regulación y promoción de la inversión que, muchas veces, llegan a saturar las capacidades de las agencias subnacionales encargadas de regular

18 «(...) Hoy día el sistema funciona así: en hora punta todo es rentable y eso subsidia las horas valla, igual pones igual flota en hora valla porque no puede poner poquísima. Entonces para que el sistema sea autosustentable la hora punta subsidia cruzadamente la hora valla. Para que un sistema funcione bien y no esté tan jodido debes poner más flota en hora punta, alguien tiene que pagar esa flota, ese es el subsidio». (Ex miembro del directorio de Protransporte)

19 Tres casos pueden ilustrar esta situación: 1) La disputa generada en 2004 entre la MML y el MTC torno a la licitación y administración de las revisiones técnicas; 2) La disputa ocurrida en 2011 entre el MTC y la MML acerca del trazo sobre el que se iba a construir la Línea 2 del metro que se cruzaba con el trazo del Metropolitano que se pensaba construir bajo la gestión de Villarán; 3) La implementación exitosa en el Callao de un sistema de control de tránsito a través de fotopapeletas a través de un consorcio peruano brasilero al mismo tiempo que en esta jurisdicción se registran serios de problemas para poder regular a las empresas prestadoras del servicio de transporte urbano regular. 
y fiscalizar el transporte ${ }^{20}$.

\section{c) Los grupos de poder en el sistema de transporte urbano}

En este eje se identificaron dos problemáticas centrales. En primer lugar, tanto los documentos de gestión consultados y los entrevistados destacaron el nivel de informalidad que tenían los prestadores del servicio de transporte. Esta situación fomentó la dispersión de sus acciones colectivas y dificultó cualquier tipo de negociación y acercamiento que pudiera tener la MML con respecto a ellas así como también le exigía a la misma que tuviera mayores consideraciones a la hora de formular compensaciones que les incentivaran a ceñirse a los requerimientos de la ley (Municipalidad Metropolitana de Lima, 2014:41).

La informalidad en el sector involucra niveles diferentes. En primer lugar, a nivel general, el crecimiento de la ciudad, el atraso del sector y la permanencia un conjunto de normas que no especificaban ni el tipo de empresa prestadora necesaria para el sector ni criterios claros de registro y autorización de conductores y rutas, facilitaron el surgimiento de modalidades de transporte alternativas no contempladas en el marco legal (colectivos) y dificultaron el efectivo control de las modalidades ya establecidas. En segundo lugar, a nivel de organizaciones comprende un amplio abanico de empresas cuyo capital de constitución es muy bajo y que las obliga a operar con un gran número de unidades que no son propias y recurren a diversas modalidades para prestar el servicio ${ }^{21}$ que implican

\footnotetext{
20 «(...) Antes todos los proyectos eran financiados por el Estado (...) A partir, digamos de (...) los años 2000 un poco antes, empezaron a sacar las iniciativas privadas, que sí es una herramienta muy poderosa para promover la ejecución de proyectos de inversión y de ahí salieron estos grandes proyectos (...) Sí, y empezaron a haber iniciativas privadas, venía un privado y te decía "Quiero sacar este proyecto y tú no lo tienes en cuenta, quiero hacerlo yo". Entonces, y salió la normativa de inversión privada. (...) Había modalidades de participación de inversión privada, concurso de proyectos integrales, licitaciones especiales, hay otras modalidades de participación en la inversión privada en proyectos públicos (...) pero el que se ha, digamos, se ha vuelto famoso y porque es rápido y es efectivo es la Iniciativa Privada (...) Lo que pasa es que la ciudad cuando crecía, cada vez se le venía un proyecto [cada vez] más grande (...) no solamente de proyectos públicos sino también proyectos privados estaban ingresando a la municipalidad. Entonces [había] ya problemas de este tipo de proyectos que no había técnicos que le ayudaran a resolver esos problemas [Resaltado del autor]» (Exfuncionario de la Gerencia de Promoción de la Inversión Privada)
}

21 «Estas empresas sin flota vehicular recurren a diversas modalidades para prestar el servicio: pueden alquilar vehículos a sus propietarios y contratar choferes y cobradores o permitir que vehículos de terceros ingresen a la ruta previo pago de sumas por cotización en razón del uso de la ruta y el seguimiento de un conjunto de 
Explorando el espacio vial: Un mapeo preliminar de los factores que influyeron en la capacidad de regulación y fiscalización del transporte urbano de la Municipalidad Metropolitana de Lima durante el período 2007-2014 - Diego Sánchez Flórez

sistemas de supervisión muy laxos por parte de la MML e incluso llegan a saltear sus marcos normativos. En tercer lugar, a nivel de los operarios, la flexibilización de los controles y el reducido precio de las tarifas permite el surgimiento de jornadas laborales extremadamente extendidas y ningún tipo de aseguramiento de los trabajadores del sector. En cuarto lugar, a nivel de las unidades de transporte, la liberalización de la importación de vehículos usados permitió que la proliferación de unidades no adecuadas para la prestación del servicio (Defensoría del Pueblo, 2008: 60-95).

En segundo lugar, los exfuncionarios destacaron el poder que tenían ciertos grupos económicos vinculados al transporte para poder influir o corromper a diversos políticos electos principalmente en el nivel provincial. En específico, se destacó que no necesariamente se trataba siempre de las mismas empresas prestadoras del servicio sino, antes bien, de un mercado de diversos actores con intereses que giraban alrededor del sector (comercializadores de vehículos, taxistas, empresas prestadoras de servicio, etc. $)^{22}$.

Dentro de estas afirmaciones, también cuatro exfuncionarios destacaron que la permeabilidad de las autoridades electas a estos intereses económicos llevaba además bastantes años arraigada en el nivel provincial y que se hacía explícita a través de la resistencia de determinados regidores y de un conjunto de medidas legales que sorpresivamente de aprobaban en el transcurso de las diversas iniciativas de regulación por parte del municipio. Estas medidas incluían acciones de amparo u otorgamientos rutas en municipios provinciales fuera de la circunscripción de la $\mathrm{MML}^{23}$.

obligaciones como la compra de combustible en determinados grifos, el uniforme, el cobro por gestión de adquisición del SOAT, entre otras». (Defensoría del Pueblo, 2008: 89).

22 «(...) El mercado del transporte urbano es un mercado de poderes (...) O sea, hay un mercado de transporte, de operadores de transporte público, hay un mercado de taxis que se ha vuelto fuerte ahora, antes no era muy fuerte el mercado de taxis, hay otro mercado por ejemplo de automóviles que hubieran volado de empresas de que venden automóviles, que tienen poder (...) O sea, si tú quieres sacar proyectos grandes tienes que saber (...) negociar, saber...implementar estrategias, porque no es fácil, no es fácil implementar un proyecto grande porque tienes que saber con quién te peleas, cómo presentas el proyecto, o sea, es un poquito complicado porque estos son mercados que tienen bastante poder (...)» (Exfuncionario de la Gerencia de Promoción de la Inversión Privada)

${ }^{23}$ «Pero [cuando] las rutas se crearon en Lima fue un sancochado que cada grupo de empresarios ponían su ruta, hacian su trámite, llegaban, "Esta es mi ruta" decían, "Cuántos buses tienes. Ya está" Y yo estuve en el primer plan regulador que era una suma de papeles canson y cada uno tenía su ruta dibujada (...) Esto se volvió 


\section{d) Falencias institucionales de la MML}

Dentro de este eje, se identificaron cuatro problemáticas. La primera de ellas se vincula al diseño institucional de la MML para poder atender y ejercer las funciones de regulación y fiscalización del transporte urbano. En ese sentido, tres exfuncionarios identificaron que la MML tiene al menos seis organismos - con distintos estatus legales- con influencia directa o indirecta para el sistema de transporte urbano. Estas son: GTU, Protransporte, EMAPE, Gerencia de Desarrollo Urbano (GDU), Servicio de Administración Tributaria (SAT), IMP, Invermet y la Gerencia de Promoción de la Inversión Privada (GPIP). Un problema que se genera como producto de esta situación es la permanente pugna que había a nivel del municipio por conseguir presupuesto. La enorme fragmentación al interior del municipio en un conjunto de agencias con atribuciones traslapadas en cuestión de transporte genera una enorme competencia por el presupuesto entre las diversas entidades que están cumpliendo objetivos comunes.

A esta situación se le sumaba que las condiciones estructurales en las que se encontraba el sector —abandonado por muchos años de la intervención estatal- hacían muy difícil reducir en corto tiempo las brechas existentes en materia de cobertura y calidad en los servicios de transporte. Todo este enorme atraso que tiene la ciudad y que las autoridades tienen que enfrentar ha venido generando una percepción en la gente que no ayudaban a facilitar ninguna reforma en el sector. En ese sentido, cuatro exfuncionarios entrevistados no dejaron de mencionar el hecho que la GTU está encargada de operar en una escala tremendamente superior a cualquier otra agencia gubernamental comprometida en el sector.

En conjunto, estos elementos llevan a que la agencia mantenga una sobrecarga de tareas tal que le impedía desempeñar efectivamente un mínimo de tareas asignadas y generaba un clima favorable para la

norma [Entrevistador: ¿En qué año fue eso?] 93, 94 (...) Pero eso es antitécnico pues ¿no? Entonces, se quiso hacer modificaciones a eso que era un desastre y obviamente los transportistas se sintieron afectados pues les ibas a quitar el negocio. Y por lo tanto buscaron congresistas, abogados para pagar acciones de amparo, se fueron a Huarochirí, se fueron al Callao y o sea, ese poder se mueve y utiliza todos los mecanismos legales y existentes para detener una propuesta como esta (...) [Entrevistador: ¿Y la gestión de Castañeda usted recuerda algún tipo de movilización así?] Siempre, de todos tipos ha habido siempre. El sector, por ejemplo, de transporte público siempre ha sido así, bien complicado». (Exfuncionario de la GPIP) 
Explorando el espacio vial: Un mapeo preliminar de los factores que influyeron en la capacidad de regulación y fiscalización del transporte urbano de la Municipalidad Metropolitana de Lima durante el período 2007-2014 - Diego Sánchez Flórez

generalización de prácticas de corrupción utilizadas por los usuarios con el fin de acelerar sus trámites. Además de ello, dado que una de las principales funciones de la GTU era otorgar autorizaciones de funcionamiento a las empresas de transporte, esta se veía permanentemente expuesta a los intereses de estos actores que buscaban mantener su actividad económica a toda costa recurriendo, en casos extremos, a sobornos.

Un segundo tipo de problemática identificada a partir de las entrevistas se vincula al organigrama mismo de la MML que no brindaba siempre las condiciones necesarias a la GTU para poder facilitarle el cumplimiento de las tareas priorizadas por su gestión. En esa línea, se destacó que este diseño organizacional no siempre tenía en cuenta la enorme carga laboral que se tenía que cubrir para poder atender a la mayor parte del sistema de transporte urbano en la ciudad ${ }^{24}$.

En tercer lugar, se identificaron serios problemas en los recursos humanos de la GTU. Tal y como lo explicaron los entrevistados y los documentos de gestión, este problema se originaba por el bajo nivel educativo general de gran parte de los trabajadores de la agencia y por la fragilidad laboral del staff de fiscalizadores. Esta última situación obligó a que el control de la MML se concentrara exclusivamente en determinadas zonas de la ciudad en desmedro de otras que requerían mayor atención y que este no pudiera tener siempre la continuidad necesaria (Defensoría del Pueblo, 2008: 140-142).

En cuarto lugar, los documentos de gestión destacan un conjunto de problemas operativos que afrontó la GTU durante el período de estudio en el desempeño de sus funciones. Estos incluían racionamiento de combustible, falta de unidades, falta de depósitos municipales, la casi total ausencia de convenios con municipios para poder implementar operativos de control y la

\footnotetext{
${ }^{24}$ Esta situación se hizo más evidente en la gestión de 2011-2014 para el caso del área legal de la GTU. En palabras de una exasesora legal: «Hay una deficiencia por ejemplo en la Municipalidad, tiene un ROF y dentro del ROF hay, algunas gerencias tienen (...) MOF creo que es (...) Si bien es cierto que la Municipalidad tiene un Área Jurídica, en específico la Municipalidad tiene 40 entre gerencias y empresas estatales, mucho mayor que un Ministerio. Entonces un Área Jurídica como la que tiene la Municipalidad no se abastecería para asesorar una Gerencia en particular con temas tan grandes como los de la GTU por ejemplo que tiene una inmensidad de temas que tiene la competencia casi de un Ministerio (...) Si bien no está propiamente escrito que deba haber una dependencia jurídica en cada Gerencia (...) sí se necesitaba un Área de Asesoría y un Área Legal».
} 
falta de un centro de control unificado del tránsito en la ciudad (Defensoría del Pueblo, 2008: 147-160). 
Explorando el espacio vial: Un mapeo preliminar de los factores que influyeron en la capacidad de regulación y fiscalización del transporte urbano de la Municipalidad Metropolitana de Lima durante el período 2007-2014 - Diego Sánchez Flórez

\section{Bibliografía}

Aguilar Villanueva, Luis F (2014). "Las dimensiones y los niveles de la gobernanza". En: Cuadernos de Gobierno Administración Pública, Vol.1, Núm. 1: 11-36. https://doi.org/10.5209/rev_cgap.2014.v1.n1.45156

Bersch, Katherine; Praca, Sergio y Taylor, Matthew (2013). "State Capacity and Bureaucratic Autonomy Within National States: Mapping the Archipielago of Excellence in Brazil". Paper preparado para ser presentado en The Latin American Studies Assosiation Conference. Washington D.C: Sin publicar. $\quad$ Recuperado de: http://www.asmetro.org.br/portal/attachments/article/1140/berschpraca-taylor-state- capacity-and-aonomy-may-1_lasa.pdf

Bielich, Claudia (2009). La Guerra del Centavo. Una mirada actual al transporte público en Lima Metropolitana. Lima: Instituto de Estudios Peruanos y Consorcio de Investigación Económica y Social

Bielich, Claudia (2009) "Perdido en el espacio (vial): El Estado en el tránsito y transporte de Lima Metropolitana". En: Tanaka, Martín (ed.) El Estado, viejo desconocido. Visiones del Estado en el Perú. Lima: IEP

Burga, César et al (1990). El Transporte Urbano de Pasajeros en Lima. Lima: Instituto de Libertad y Democracia

Dargent, Eduardo (2012). El Estado en el Perú. Una Agenda de Investigación. Lima: PUCP

De Soto, Hernando (1989). El Otro Sendero. Lima: Instituto de Libertad y Democracia 
Defensoría del Pueblo (2008). “Informe Defensorial Nº137: Transporte

Urbano en Lima Metropolitana:Un desafío a la defensa de la Vida". Recuperado de:

http://www.defensoria.gob.pe/modules/Downloads/informes/defenso -riales/informe_137.pdf

Figueroa, Oscar (2005). “Transporte Urbano y Globalización. Políticas y Efectos en América Latina”. En: Revista Eure Vol. XXXI n ${ }^{\circ} 94-$ Santiago de Chile. Pp. 41-53. https://doi.org/10.4067/s0250$\underline{71612005009400003}$

Fukuyama, Francis (2004). "Elementos ausentes en la estatalidad". En:

La Construcción del Estado. Hacia un nuevo orden mundial en el s. XXI. Buenos Aires: Ediciones B.

Glave, Marisa (2016). Aciertos y Limitaciones de una Experiencia de Gestión. Lima: Instituto de Estudios Peruanos

Lindblom, Charles E. El proceso de elaboración de políticas públicas. México D.F: Ministerio para las Administraciones Públicas

Municipalidad Metropolitana De Lima (2014). Lima lo hizo. Informe de gestión 2011/2014

Muñoz, Paula (2005). El diseño institucional municipal 1980-2004 y sus implicancias para las zonas rurales. Lima: Asociación Servicios Educativos Rurales

Repetto, Fabián (2004). "Capacidad Estatal: requisito necesario para una mejor política social en América Latina". Panamá, VIII Congreso Internacional del CLAD sobre Reforma del Estado y de la Administración Pública. Recuperado de: http://idbdocs.iadb.org/wsdocs/getdocument.aspx?docnum=2220291 
Explorando el espacio vial: Un mapeo preliminar de los factores que influyeron en la capacidad de regulación y fiscalización del transporte urbano de la Municipalidad Metropolitana de Lima durante el período 2007-2014 - Diego Sánchez Flórez

Skocpol, Theda (1989). "El Estado regresa al primer plano: Estrategias de análisis en la investigación actual”. En: Lecturas sobre el Estado y las Políticas Públicas: Retomando el Debate de Ayer para Fortalecer el Actual. Buenos Aires: Proyecto de Modernización del EstadoJefatura de Ministros de la Nación.

Soifer, Hillel (2015). State Building in Latin America. Cambridge University Press. https://doi.org/10.1017/cbo9781316257289

Zavala, Fernando (1995). "La privatización del servicio de transporte urbano en Lima Metropolitana". En: Ortiz De Zevallos, Gabriel \& Alvarez Rodrich, Augusto (ed.) La Implementación de Políticas Públicas en el Perú. Lima: Apoyo. Pp. 116-129 